\title{
Harmonic mappings in the exterior of the unit disk
}

\begin{abstract}
In this paper we consider a class of univalent orientation-preserving harmonic functions defined on the exterior of the unit disk which satisfy the condition $\sum_{n=1}^{\infty} n^{p}\left(\left|a_{n}\right|+\left|b_{n}\right|\right) \leq 1$. We are interested in finding radius of univalence and convexity for such class and we find extremal functions. Convolution, convex combination, and explicit quasiconformal extension for this class are also determined.
\end{abstract}

1. Introduction. Let $\Sigma_{H}$ be the family of complex-valued harmonic, orientation-preserving, univalent mappings of the form

$$
f(z)=h(z)+\overline{g(z)}
$$

defined on $\tilde{\mathbb{U}}=\{z:|z|>1\}$, where

$$
h(z)=z+\sum_{n=1}^{\infty} a_{n} z^{-n} \quad \text { and } \quad g(z)=\sum_{n=1}^{\infty} b_{n} z^{-n}
$$

are analytic in $\tilde{\mathbb{U}}$ and coefficients $a_{n}, b_{n}$ are complex. A necessary and sufficient condition for $f$ to be locally univalent and sense-preserving in $\tilde{\mathbb{U}}$ is that $\left|g^{\prime}(z)\right|<\left|h^{\prime}(z)\right|$ in $\tilde{\mathbb{U}}$.

Let $\Sigma_{H}^{*}(\alpha)$ and $\Sigma_{C H}(\beta)$ be the subclasses of $\Sigma_{H}$ consisting of functions $f$ that are starlike of order $\alpha, 0 \leq \alpha<1$, and convex of order $\beta, 0 \leq \beta<1$,

2000 Mathematics Subject Classification. 30C45.

Key words and phrases. Harmonic mapping, meromorphic, quasiconformal extension, radius of convexity, radius of univalence. 
respectively. It is obvious that for $0<\alpha<1$ and $0<\beta<1, \Sigma_{H}^{*}(\alpha) \subset \Sigma_{H}^{*}(0)$ and $\Sigma_{C H}(\beta) \subset \Sigma_{C H}(0)$.

We say that $f \in \Sigma_{H}$ is harmonic starlike of order $\alpha, 0 \leq \alpha<1$, in $\tilde{\mathbb{U}}$, if $f \neq 0$ and

$$
\frac{\partial}{\partial \theta}\left(\arg f\left(r e^{i \theta}\right)\right) \geq \alpha, \quad 0 \leq \theta<2 \pi,
$$

for each $z,|z|=r>1$. For the Jordan curve this condition means that $f$ is starlike with respect to the origin.

A function $f \in \Sigma_{H}$ is harmonic convex of order $\beta, 0 \leq \beta<1$, in $\tilde{\mathbb{U}}$, if

$$
\frac{\partial}{\partial \theta}\left\{\arg \left(\frac{\partial}{\partial \theta} f\left(r e^{i \theta}\right)\right)\right\} \geq \beta
$$

for each $z,|z|=r>1$.

We further denote by $\mathcal{T}_{H}^{*}(\alpha)$ and $\mathcal{T}_{C H}(\beta)$ the subclasses of $\Sigma_{H}^{*}(\alpha)$ and $\Sigma_{C H}(\beta)$, respectively, consisting of functions $f=h+\bar{g}$ so that $h$ and $g$ are of the form

(2) $h(z)=z+\sum_{n=1}^{\infty} a_{n} z^{-n}$ and $g(z)=-\sum_{n=1}^{\infty} b_{n} z^{-n}, \quad$ where $\quad a_{n}, b_{n} \geq 0$.

In 1985 Hengartner and Schober [1] studied the class $\Sigma_{H}$ and proved that it is compact with respect to the topology of locally uniform convergence. They showed that if $f \in \Sigma_{H}$, then $\left|b_{1}\right| \leq 1$ and $\left|b_{2}\right| \leq \frac{1}{2}\left(1-\left|b_{1}\right|^{2}\right) \leq \frac{1}{2}$ and the coefficient bounds are sharp. In the first case the extremal function has the form $f(z)=z+\frac{b_{1}}{\bar{z}}$ and maps $\tilde{\mathbb{U}}$ onto the exterior possibly degenerated circle $|w|=2 \cos \left(\frac{\gamma}{2}\right), \gamma \in[-\pi, \pi], b_{1}=e^{i \gamma}$, while in the second case the extremal function is of the form $f(z)=z+\frac{1}{2 \bar{z}^{2}}$ and maps $\tilde{\mathbb{U}}$ onto the complement of the cardioid. Moreover, they proved that if $f \in \Sigma_{H}$, then $\sum_{n=1}^{\infty} n\left(\left|a_{n}\right|^{2}-\left|b_{n}\right|^{2}\right) \leq 1+2 \operatorname{Re} b_{1}$. This result is sharp and equality occurs if and only if the set $\mathbb{C} \backslash f(\tilde{\mathbb{U}})$ has area zero.

In 1999 Jahangiri and Silverman [3], among other things, proved that if $f=h+\bar{g}$, where $h$ and $g$ are of the form (1) and satisfy the condition $\sum_{n=1}^{\infty} n\left(\left|a_{n}\right|+\left|b_{n}\right|\right) \leq 1$, then $f$ is orientation preserving and univalent in $\tilde{\mathbb{U}}$. They also showed that this condition is sufficient for functions $f=h+\bar{g}$ to be in $\Sigma_{H}^{*}(0)$, and sufficient and necessary for the subclass $\mathcal{T}_{H}^{*}(0)$. We have to remark that the authors skipped the assumption $f \neq 0$ in the determination of the class. In [2] Jahangiri extended these results to the general case $\Sigma_{H}^{*}(\alpha)$, $0 \leq \alpha<1$. He proved the following theorems which we shall use in this paper.

Theorem A. Let $f=h+\bar{g}$ be of the forms (1). If

$$
\sum_{n=1}^{\infty}\left(\frac{n+\alpha}{1-\alpha}\left|a_{n}\right|+\frac{n-\alpha}{1-\alpha}\left|b_{n}\right|\right) \leq 1, \quad 0 \leq \alpha<1,
$$


then $f$ is harmonic, orientation preserving, univalent in $\tilde{\mathbb{U}}$ and $f \in \Sigma_{H}^{*}(\alpha)$.

Theorem B. A sufficient condition for $f=h+\bar{g}$ of the forms (1) to belong to $\Sigma_{C H}(\beta), 0 \leq \beta<1$, is that

$$
\sum_{n=1}^{\infty}\left(\frac{n(n+\beta)}{1-\beta}\left|a_{n}\right|+\frac{n(n-\beta)}{1-\beta}\left|b_{n}\right|\right) \leq 1 .
$$

He showed that above conditions are also necessary when the coefficients of $h$ and $g$ are restricted by (2). Furthermore, in [2] the extreme points for $\mathcal{T}_{H}^{*}(\alpha)$ are also characterized and the closure properties under convolution and convex combination are proved.

2. Main results. Consider harmonic functions $f: \tilde{\mathbb{U}} \rightarrow \overline{\mathbb{C}}$ of the form

$$
f(z)=z+\sum_{n=1}^{\infty} a_{n} z^{-n}+\overline{\sum_{n=1}^{\infty} b_{n} z^{-n}}
$$

and suppose that they satisfy the condition

$$
\sum_{n=1}^{\infty} n^{p}\left(\left|a_{n}\right|+\left|b_{n}\right|\right) \leq 1
$$

Then $|f(z)| \geq|z|-\frac{1}{|z|}, z \in \tilde{\mathbb{U}}$. This shows that in the exterior of the unit disk $f \neq 0$. Hence, if $f \in \Sigma_{H}$ provided (4), then $f$ is univalent and 0 is in the complement of the image of $\tilde{\mathbb{U}}$.

We will try to find the relation between the condition (4) (with some $p>0$ ) and properties of the harmonic function (3).

Theorem 1. If harmonic function $f: \tilde{\mathbb{U}} \rightarrow \overline{\mathbb{C}}$ of the form (3), with $a_{1}=0$ satisfies the condition (4) for some $p \geq 1$, then $f \in \Sigma_{H}^{*}(\alpha)$, where $\alpha=$ $\alpha(p)=\frac{2^{p}-2}{2^{p}+1}$.

Proof. Let $f$ be of the form (3). According to (4) we have

$$
\begin{aligned}
\left|h^{\prime}(z)\right| \geq & -\sum_{n=1}^{\infty} n\left|a_{n}\right||z|^{-n-1}>1-\sum_{n=1}^{\infty} n\left|a_{n}\right| \geq 1-\sum_{n=1}^{\infty} n^{p}\left|a_{n}\right| \\
& \geq \sum_{n=1}^{\infty} n^{p}\left|b_{n}\right| \geq \sum_{n=1}^{\infty} n\left|b_{n}\right|>\sum_{n=1}^{\infty} n\left|b_{n}\right||z|^{-n-1} \geq\left|g^{\prime}(z)\right| .
\end{aligned}
$$

Hence $f$ is a sense-preserving mapping.

By Theorem A and condition (4), $f$ will be harmonic starlike of order $\alpha=\alpha(p)$, if

$$
n^{p} \geq \frac{n+\alpha}{1-\alpha}, \quad n=2,3, \ldots
$$


The above expression may be written as

$$
\alpha \leq \frac{n^{p}-n}{n^{p}+1}, \quad n=2,3, \ldots
$$

It is sufficient to show that $g_{p}(n)=\frac{n^{p}-n}{n^{p}+1}$ (with a fixed $p \geq 1$ and $n=$ $2,3, \ldots)$ is an increasing sequence and hence $\frac{n^{p}-n}{n^{p}+1} \geq \frac{2^{p}-2}{2^{p}+1}=g_{p}(2)=\alpha(p)$.

Remark 1. If $a_{1} \neq 0$, then Theorem 1 is valid only for $\alpha=0$.

It is obvious that if $\sum_{n=1}^{\infty} n^{p}\left(\left|a_{n}\right|+\left|b_{n}\right|\right) \leq 1$ and $p \geq 2$, then $f$ is a harmonic convex of order 0 . Note that if $p \in[1,2)$, then $f$ needn't be a convex harmonic function in $\tilde{\mathbb{U}}$. For example, let us consider the function $f_{p}(z)=z-\frac{1}{2^{p}} \frac{1}{\bar{z}^{2}}$. We have

$$
\begin{aligned}
\frac{\partial}{\partial \theta}\left\{\arg \left\{\frac{\partial}{\partial \theta} f\left(r e^{i \theta}\right)\right\}\right\} & =\operatorname{Re}\left\{\frac{z h^{\prime}(z)+z^{2} h^{\prime \prime}(z)+\overline{z g^{\prime}(z)+z^{2} g^{\prime \prime}(z)}}{z h^{\prime}(z)-\overline{z g^{\prime}(z)}}\right\} \\
& =\operatorname{Re}\left\{\frac{z-\frac{1}{2^{p-2}} \frac{1}{\bar{z}^{2}}}{z-\frac{1}{2^{p-1}} \frac{1}{\bar{z}^{2}}}\right\} .
\end{aligned}
$$

It is convenient to choose the values of $z$ on the positive real axis where $z=r>1$. Under this additional assumption, we see that the numerator is negative for $1<r \leq \sqrt[3]{\frac{1}{2^{p-2}}}$. Thus the quotient is negative and $f_{p}$ does not belong to $\Sigma_{C H}(0)$. A simple calculations show that for $|z|=1, f_{p}$ does not belong to $\Sigma_{C H}(0)$ too.

Theorem 2. If harmonic function $f: \tilde{\mathbb{U}} \rightarrow \overline{\mathbb{C}}$ of the form (3) satisfies the condition (4) for some $1 \leq p<2$, then $f$ maps $\Delta_{p}=\left\{z:|z|>\sqrt[5]{4^{2-p}}\right\}$ onto a domain which complement is convex. The result is sharp with extremal functions $f_{1}(z)=z-\frac{1}{4^{p}} \frac{1}{z^{4}}$ and $f_{2}(z)=z-\frac{1}{4^{p}} \frac{1}{\bar{z}^{4}}$.

Proof. Our proof starts with the observation that if $p \geq 1$, then

$$
\sum_{n=1}^{\infty} n\left(\left|a_{n}\right|+\left|b_{n}\right|\right) \leq \sum_{n=1}^{\infty} n^{p}\left(\left|a_{n}\right|+\left|b_{n}\right|\right) \leq 1
$$

and then $f \in \Sigma_{H}^{*}(0)$ (see Theorem A). For $z \in \tilde{\mathbb{U}}$ and $R \geq 1$ we have

$$
\frac{1}{R} f(R z)=z+\sum_{n=1}^{\infty} \frac{a_{n}}{R^{n+1} z^{n}}+\overline{\sum_{n=1}^{\infty} \frac{b_{n}}{R^{n+1} z^{n}} .}
$$

From this we conclude that

$$
\sum_{n=1}^{\infty} \frac{n^{2}}{R^{n+1}}\left(\left|a_{n}\right|+\left|b_{n}\right|\right) \leq \sum_{n=1}^{\infty} n^{p}\left(\left|a_{n}\right|+\left|b_{n}\right|\right) \leq 1
$$


if $\frac{n^{2}}{R^{n+1}} \leq n^{p}$ or equivalently $R \geq \sqrt[n+1]{n^{2-p}}, n=2,3, \ldots$. It remains to find the maximal value of the right-hand of previous inequality. Considering the function $f(x)=e^{\frac{2-p}{x+1} \ln x}$ for a fixed $1 \leq p<2$ and $x \geq 2$, we obtain that $R=R(p)=\sup _{n \in \mathbb{N}} \sqrt[n+1]{n^{2-p}}=\sqrt[5]{4^{2-p}}$. Easy computation shows that the extremal functions are $f_{1}(z)=z-\frac{1}{4^{p}} \frac{1}{z^{4}}$ and $f_{2}(z)=z-\frac{1}{4^{p}} \frac{1}{\bar{z}^{4}}$.

Let us mention an interesting consequence of this theorem.

Corollary 1. If $\sum_{n=1}^{\infty} n\left(\left|a_{n}\right|+\left|b_{n}\right|\right) \leq 1$, then $f$ given by (3) maps $\{z$ : $|z|>\sqrt[5]{4}\}$ onto a domain which complement is convex.

Theorem 3. If harmonic function $f: \tilde{\mathbb{U}} \rightarrow \overline{\mathbb{C}}$ of the form (3), with $a_{1}=0$ satisfies the condition (4) for some $p \geq 2$, then $f \in \Sigma_{C H}(\beta)$, where $\beta=$ $\beta(p)=\frac{2^{p}-4}{2^{p}+2}$.

Proof. Note that

$$
\begin{aligned}
\sum_{n=1}^{\infty}\left(\frac{n(n+\beta)}{1-\beta}\left|a_{n}\right|+\frac{n(n-\beta)}{1-\beta}\left|b_{n}\right|\right) & \leq \sum_{n=1}^{\infty} \frac{n(n+\beta)}{1-\beta}\left(\left|a_{n}\right|+\left|b_{n}\right|\right) \\
& \leq \sum_{n=1}^{\infty} n^{p}\left(\left|a_{n}\right|+\left|b_{n}\right|\right) \leq 1
\end{aligned}
$$

whenever

$$
\frac{n(n+\beta)}{1-\beta} \leq n^{p}
$$

or equivalently

$$
\beta \leq \frac{n^{p}-n^{2}}{n^{p}+n}=g_{p}(n), \quad n=2,3, \ldots
$$

What is left, is to show that the right-hand side of the above inequality is an increasing function of variable $n$. Hence

$$
\frac{n^{p}-n^{2}}{n^{p}+n}=g_{p}(n) \geq \frac{2^{p}-4}{2^{p}+2}=g_{p}(2)=\beta(p)
$$

and then $f \in \Sigma_{C H}(\beta)$ (see Theorem B).

Remark 2. If $a_{1} \neq 0$, then Theorem 3 is valid only for $\beta=0$.

The following theorem yields information about properties of the harmonic functions of the form (3) under assumption (4) for $0<p \leq 1$.

Theorem 4. Let $f$ be of the form (3) and satisfies (4) for some $0<p \leq 1$. Then $f$ is univalent and sense-preserving harmonic map in $\Delta_{p+1}=\{z$ : $\left.|z|>\sqrt[5]{4^{1-p}}\right\}$. Moreover, $f$ maps

(i) $\Delta_{p+1}$ onto a domain which complement is starlike,

(ii) $\Delta_{p}$ onto a domain which complement is convex. 
The results are sharp with extremal functions $f_{1}(z)=z-\frac{1}{4^{p}} \frac{1}{z^{4}}$ and $f_{2}(z)=$ $z-\frac{1}{4^{p}} \frac{1}{\bar{z}^{4}}$.

Proof. We begin by proving that $f$ is locally univalent and sense-preserving in the domain $\Delta_{p+1}$. Let $h$ and $g$ are of the form (1), then for $|z|>\sqrt[5]{4^{1-p}}$ and $n=1,2, \ldots$ we have

$$
\begin{aligned}
\left|h^{\prime}(z)\right| \geq & 1-\sum_{n=1}^{\infty} n\left|a_{n}\right||z|^{-n-1}>1-\sum_{n=1}^{\infty} n\left|a_{n}\right|\left(4^{\frac{(p-1)(n+1)}{5}}\right) \geq 1-\sum_{n=1}^{\infty} n^{p}\left|a_{n}\right| \\
& \geq \sum_{n=1}^{\infty} n^{p}\left|b_{n}\right| \geq \sum_{n=1}^{\infty} n\left|b_{n}\right|\left(4^{\frac{(p-1)(n+1)}{5}}\right)>\sum_{n=1}^{\infty} n\left|b_{n}\right||z|^{-n-1} \geq\left|g^{\prime}(z)\right| .
\end{aligned}
$$

This is because

$$
n \cdot 4^{\frac{(p-1)(n+1)}{5}} \leq n^{p}, \quad n=1,2, \ldots
$$

Therefore $f$ is locally univalent and sense-preserving in $\Delta_{p+1}$. To show that $f$ is univalent in $\Delta_{p+1}$, we notice that if $g(z) \equiv 0$, then $f_{1}^{*}(z)=$ $4^{\frac{p-1}{5}} h\left(4^{\frac{1-p}{5}} z\right)=z+\sum_{n=1}^{\infty} a_{n} 4^{\frac{(p-1)(n+1)}{5}} z^{-n}$ is analytic and the univalence of $f$ comes from starlikeness and (5).

Let $g(z) \neq 0$ and $0<p \leq 1$. We show that if $z_{1}, z_{2} \in \Delta_{p+1}$ and $z_{1} \neq z_{2}$, then $f\left(z_{1}\right) \neq f\left(z_{2}\right)$. We can write

$$
\begin{array}{r}
\left|\frac{f\left(z_{1}\right)-f\left(z_{2}\right)}{z_{1}-z_{2}}\right|=\mid \frac{z_{1}-z_{2}+\sum_{n=1}^{\infty} a_{n} \frac{z_{2}^{n}-z_{1}^{n}}{\left(z_{1} z_{2}\right)^{n}}+\sum_{n=1}^{\infty} \overline{b_{n}} \frac{\overline{z_{2}^{n}-z_{1}^{n}}}{z_{1}-z_{2}} \mid}{\geq 1-\sum_{n=1}^{\infty} n \frac{\left(\left|a_{n}\right|+\left|b_{n}\right|\right)}{\left|z_{1}\right|\left|z_{2}\right|^{n}}>1-\sum_{n=1}^{\infty} n\left(\left|a_{n}\right|+\left|b_{n}\right|\right)\left(4^{\frac{(p-1)(n+1)}{5}}\right)} \\
\geq 1-\sum_{n=1}^{\infty} n^{p}\left(\left|a_{n}\right|+\left|b_{n}\right|\right) \geq 0 .
\end{array}
$$

Thus $f\left(z_{1}\right) \neq f\left(z_{2}\right)$. Our next claim is to show that $f$ is starlike in $\Delta_{p+1}$. Let $z \in \Delta_{p+1}$ then

$$
f_{2}^{*}(z)=4^{\frac{p-1}{5}} f\left(4^{\frac{1-p}{5}} z\right)=z+\sum_{n=1}^{\infty}\left(a_{n} z^{-n}+\overline{b_{n} z^{-n}}\right) 4^{\frac{(p-1)(n+1)}{5}}
$$

and by (5)

$$
\sum_{n=1}^{\infty} 4^{\frac{(p-1)(n+1)}{5}} n\left(\left|a_{n}\right|+\left|b_{n}\right|\right) \leq \sum_{n=1}^{\infty} n^{p}\left(\left|a_{n}\right|+\left|b_{n}\right|\right) \leq 1 .
$$


Hence $f$ is starlike in $\Delta_{p+1}$. Furthermore, $f$ is convex in $\Delta_{p}=\{z:|z|>$ $\left.4^{\frac{2-p}{5}}\right\}$ and $\Delta_{p} \subset \Delta_{p+1}$. To see this we can consider the function

$$
f_{3}^{*}(z)=4^{\frac{p-2}{5}} f\left(4^{\frac{2-p}{5}} z\right)=z+\sum_{n=1}^{\infty}\left(a_{n} z^{-n}+\overline{b_{n} z^{-n}}\right) 4^{\frac{(p-2)(n+1)}{5}} .
$$

This ends the proof.

Theorem 5. Let $f$ be of the form (3) and satisfies (4) for some $0<p<\infty$. Then

$$
\left\{w:|w|>4^{\frac{1-p}{5}}+4^{\frac{p-1}{5}}\right\} \subset f\left(\Delta_{p+1}\right) .
$$

Proof. Let $w=f\left(r e^{i \theta}\right), r \geq 1$. Then for $p>0$ we have

$$
\begin{aligned}
\left|f\left(r e^{i \theta}\right)\right| \leq r+\sum_{n=1}^{\infty}\left|a_{n}\right| r^{-n}+\sum_{n=1}^{\infty}\left|b_{n}\right| r^{-n} & =r+\frac{1}{r} \sum_{n=1}^{\infty}\left(\left|a_{n}\right|+\left|b_{n}\right|\right) r^{-n+1} \leq \\
r & +\frac{1}{r} \sum_{n=1}^{\infty} n^{p}\left(\left|a_{n}\right|+\left|b_{n}\right|\right) \leq r+\frac{1}{r} .
\end{aligned}
$$

By Theorem 4, if $p>0$ then $f$ is harmonic univalent in $\Delta_{p+1}$. Letting $r \rightarrow 4^{\frac{1-p}{5}}$ in the above inequality, the result follows.

Corollary 2. If $\sum_{n=1}^{\infty} n\left(\left|a_{n}\right|+\left|b_{n}\right|\right) \leq 1$, then $\{w:|w|>2\} \subset f(\tilde{\mathbb{U}})$.

3. Convolution. For harmonic functions $f_{1}$ and $f_{2}$ of the form

(6) $f_{1}(z)=z+\sum_{n=1}^{\infty} a_{n} z^{-n}+\sum_{n=1}^{\infty} \overline{b_{n} z^{-n}}, \quad f_{2}(z)=z+\sum_{n=1}^{\infty} A_{n} z^{-n}-\sum_{n=1}^{\infty} B_{n} \overline{z^{-n}}$,

where $A_{n}, B_{n} \geq 0$, we define the convolution of $f_{1}$ and $f_{2}$ as

$$
\left(f_{1} * f_{2}\right)(z)=z+\sum_{n=1}^{\infty} a_{n} A_{n} z^{-n}+\sum_{n=1}^{\infty}\left(-\overline{b_{n}} B_{n}\right) \overline{z^{-n}} .
$$

In particular, if $f=h+\bar{g}$ with $h$ and $g$ of the form (1), then

$$
\left(f_{1} * f_{2}\right)(z)=z+\sum_{n=1}^{\infty} a_{n} A_{n} z^{-n}+\sum_{n=1}^{\infty} \overline{b_{n} B_{n} z^{-n}} .
$$

Theorem 6. Suppose that $\sum_{n=1}^{\infty} n^{p}\left(\left|a_{n}\right|+\left|b_{n}\right|\right) \leq 1, p>0$, and $f_{1}, f_{2}$ are of the form (6). Then

(i) if $f_{2} \in \mathcal{T}_{H}^{*}(\alpha)$, then $f_{1} * f_{2} \in \Sigma_{H}^{*}(\alpha)$,

(ii) if $f_{2} \in \mathcal{T}_{C H}(\beta)$, then $f_{1} * f_{2} \in \Sigma_{C H}(\beta)$. 
Proof. We justify only the case (ii). Note that if $\sum_{n=1}^{\infty} n^{p}\left(\left|a_{n}\right|+\left|b_{n}\right|\right) \leq 1$, $p>0$, then $\left|a_{n}\right| \leq 1$ and $\left|b_{n}\right| \leq 1, n=1,2, \ldots$. From this we conclude that

$$
\begin{aligned}
\sum_{n=1}^{\infty}\left(\frac{n(n+\beta)}{1-\beta}\left|a_{n}\right| A_{n}+\frac{n(n-\beta)}{1-\beta}\left|b_{n}\right| B_{n}\right) \\
\leq \sum_{n=1}^{\infty}\left(\frac{n(n+\beta)}{1-\beta} A_{n}+\frac{n(n-\beta)}{1-\beta} B_{n}\right) \leq 1 .
\end{aligned}
$$

This is because the coefficient condition $\sum_{n=1}^{\infty}\left(\frac{n(n+\beta)}{1-\beta} A_{n}+\frac{n(n-\beta)}{1-\beta} B_{n}\right) \leq$ 1 is necessary for functions $f_{2}$ to be in the class $\mathcal{T}_{C H}(\beta)$ (see [2]). Hence $f_{1} * f_{2} \in \Sigma_{C H}(\beta)$.

Now we are going to examine the convolution properties of the functions $f_{1}$ and $f_{2}$ of the form (6) under assumptions $\sum_{n=1}^{\infty} n^{p}\left(\left|a_{n}\right|+\left|b_{n}\right|\right) \leq 1$ and $\sum_{n=1}^{\infty} n^{q}\left(\left|A_{n}\right|+\left|B_{n}\right|\right) \leq 1, p>0, q>0$.

Theorem 7. If $\sum_{n=1}^{\infty} n^{p}\left(\left|a_{n}\right|+\left|b_{n}\right|\right) \leq 1$ and $\sum_{n=1}^{\infty} n^{q}\left(\left|A_{n}\right|+\left|B_{n}\right|\right) \leq 1$, $p+q \geq 1$, then

$$
F(z)=z+\sum_{n=2}^{\infty} a_{n} A_{n} z^{-n}+\sum_{n=1}^{\infty} \overline{b_{n} B_{n} z^{-n}} \in \Sigma_{H}^{*}\left(\frac{2^{p+q}-2}{2^{p+q}+1}\right) .
$$

Proof. Assume that $F(z)=z+\sum_{n=2}^{\infty} a_{n} A_{n} z^{-n}+\sum_{n=1}^{\infty} \overline{b_{n} B_{n} z^{-n}}$. It is clear that

$$
\begin{aligned}
\sum_{n=1}^{\infty} n^{p+q} & \left(\left|a_{n} A_{n}\right|+\left|b_{n} B_{n}\right|\right) \\
& \leq\left(\sum_{n=1}^{\infty} n^{p}\left(\left|a_{n}\right|+\left|b_{n}\right|\right)\right)\left(\sum_{n=1}^{\infty} n^{q}\left(\left|A_{n}\right|+\left|B_{n}\right|\right)\right) \leq 1 .
\end{aligned}
$$

By Theorem 1 we have that $F \in \Sigma_{H}^{*}\left(\frac{2^{p+q}-2}{2^{p+q}+1}\right)$.

Taking $p=1$ and $q=2$ in the above theorem, we obtain

Corollary 3. If $f_{1} \in \mathcal{T}_{H}^{*}(0)$ and $f_{2} \in \mathcal{T}_{C H}(0)$, with $a_{1}=A_{1}=0$, then $f_{1} * f_{2} \in \Sigma_{H}^{*}\left(\frac{2}{3}\right)$.

In the same way as Theorem 7 we can prove the following result.

Theorem 8. If $\sum_{n=1}^{\infty} n^{p}\left(\left|a_{n}\right|+\left|b_{n}\right|\right) \leq 1$ and $\sum_{n=1}^{\infty} n^{q}\left(\left|A_{n}\right|+\left|B_{n}\right|\right) \leq 1$, $p+q \geq 2$, then

$$
F(z)=z+\sum_{n=2}^{\infty} a_{n} A_{n} z^{-n}+\sum_{n=1}^{\infty} \overline{b_{n} B_{n} z^{-n}} \in \Sigma_{C H}\left(\frac{2^{p+q}-4}{2^{p+q}+2}\right) .
$$

Substituting $p=q=1$ in the Theorem 8 , we have 
Corollary 4. If $f_{1}$ and $f_{2}$ belong to the class $\mathcal{T}_{H}^{*}(0)$, with $a_{1}=A_{1}=0$, then $f_{1} * f_{2} \in \Sigma_{C H}(0)$.

\section{Quasiconformal extension of harmonic meromorphic mappings.}

It is well known that the condition (4) does not imply the possibility of quasiconformal extension of $f$.

Consider the class of functions $f$ of the form (3) that satisfy, for some $p \geq 1$, the condition

$$
\sum_{n=1}^{\infty} n^{p}\left(\left|a_{n}\right|+\left|b_{n}\right|\right) \leq k, \quad k \in(0,1) .
$$

A necessary condition for a harmonic mapping $f$ to have a quasiconformal extension to the whole plane is the following: the image $f(\mathbb{T})$ is a quasicircle, where $\mathbb{T}=\{z \in \mathbb{C}:|z|=1\}$.

Recall that a Jordan curve is a quasicircle, if it is a homeomorphic image of a unit circumference under a quasiconformal mapping of the extended plane $\overline{\mathbb{C}}$ onto itself.

We first prove:

Lemma. Suppose that $f$ of the form (3) satisfies the condition (7). Then the curve $f(\mathbb{T})$ is a quasicircle.

Proof. Let us first observe that for $f=h+\bar{g}$, where $h$ and $g$ are of the form (1), we have

$$
\begin{aligned}
\left|\omega_{f}(z)\right| & =\left|\frac{g^{\prime}(z)}{h^{\prime}(z)}\right| \leq \frac{\sum_{n=1}^{\infty} n \frac{\left|b_{n}\right|}{|z|^{n+1}}}{1-\sum_{n=1}^{\infty} n \frac{\left|a_{n}\right|}{|z|^{n+1}}} \leq \frac{\sum_{n=1}^{\infty} n^{p}\left|b_{n}\right|}{1-\sum_{n=1}^{\infty} n^{p}\left|a_{n}\right|} \\
& \leq \frac{\sum_{n=1}^{\infty} n^{p}\left|b_{n}\right|}{1-k+\sum_{n=1}^{\infty} n^{p}\left|b_{n}\right|}=1-\frac{1-k}{1-k+\sum_{n=1}^{\infty} n^{p}\left|b_{n}\right|} \leq k .
\end{aligned}
$$

For $z_{1}, z_{2} \in \tilde{\mathbb{U}}$ such that $z_{1} \neq z_{2}$ we have

$$
\begin{array}{r}
\left|f\left(z_{1}\right)-f\left(z_{2}\right)\right|=\left|z_{1}-z_{2}+\sum_{n=1}^{\infty} a_{n}\left(z_{1}^{-n}-z_{2}^{-n}\right)+\overline{\sum_{n=1}^{\infty} b_{n}\left(z_{1}^{-n}-z_{2}^{-n}\right)}\right| \\
(8) \leq\left|z_{1}-z_{2}\right|\left(1+\sum_{n=1}^{\infty}\left(\left|a_{n}\right|+\left|b_{n}\right|\right) \frac{\left|z_{2}^{n-1}+z_{2}^{n-2} z_{1}+\ldots+z_{1}^{n-1}\right|}{\left|z_{1} z_{2}\right|^{n}}\right) \\
<\left|z_{1}-z_{2}\right|\left(1+\sum_{n=1}^{\infty} n^{p}\left(\left|a_{n}\right|+\left|b_{n}\right|\right)\right)<\left|z_{1}-z_{2}\right|(1+k) .
\end{array}
$$

Moreover,

$$
\begin{aligned}
\left|f\left(z_{1}\right)-f\left(z_{2}\right)\right| & \geq\left|z_{1}-z_{2}\right|\left(1-\sum_{n=1}^{\infty} n^{p}\left(\left|a_{n}\right|+\left|b_{n}\right|\right)\right) \\
& >\left|z_{1}-z_{2}\right|(1-k)>0 .
\end{aligned}
$$


From this, we conclude that $f$ has homeomorphic extension on $\overline{\widetilde{U}}$, which also satisfies (8) and (9). Therefore, the image line $\Gamma=f(\mathbb{T})$ is a Jordan curve. According to Pommerenke (see [5], Lemma 9.8), a Jordan curve $\gamma$ is a quasicircle if and only if

$$
K(\gamma)=\left|\frac{w_{1}-w_{3}}{w_{2}-w_{3}}\right|\left|\frac{w_{2}-w_{4}}{w_{1}-w_{4}}\right| \geq \delta>0,
$$

for some positive constant $\delta$ and for all cyclically ordered points $w_{1}, w_{2}, w_{3}$, $w_{4}$ on $\gamma$. We deduce from (8) and (9) that

$$
K(\Gamma) \geq\left(\frac{1-k}{1+k}\right)^{2} \cdot K(\mathbb{T})>0
$$

and finally that $f(\mathbb{T})$ is a quasicircle.

Theorem 9. Let $f$ be of the form (3) and satisfies the condition (7). Then the mapping

$$
F(z)= \begin{cases}f(z), & |z| \geq 1 \\ z+\sum_{n=1}^{\infty} a_{n} \overline{z^{n}}+\sum_{n=1}^{\infty} \overline{b_{n}} z^{n}, & |z| \leq 1\end{cases}
$$

is a quasiconformal extension of $f$ onto $\overline{\mathbb{C}}$. Moreover, its complex dilatation $\mu_{F}$ satisfies $\left|\mu_{F}(z)\right|<k$.

Proof. Our proof starts with the observation that for $z_{1}, z_{2} \in \mathbb{C} \backslash \overline{\tilde{U}}$ such that $z_{1} \neq z_{2}$ we obtain

$$
\begin{aligned}
\left|f\left(z_{1}\right)-f\left(z_{2}\right)\right| & =\left|z_{1}-z_{2}+\sum_{n=1}^{\infty} a_{n}\left(\overline{z_{1}^{n}}-\overline{z_{2}^{n}}\right)+\sum_{n=1}^{\infty} \overline{b_{n}}\left(z_{1}^{n}-z_{2}^{n}\right)\right| \\
& \leq\left|z_{1}-z_{2}\right|\left(1+\sum_{n=1}^{\infty}\left(\left|a_{n}\right|+\left|b_{n}\right|\right)\left|z_{1}^{n-1}+z_{1}^{n-2} z_{2}+\ldots z_{2}^{n-1}\right|\right) \\
& <\left|z_{1}-z_{2}\right|\left(1+\sum_{n=1}^{\infty} n^{p}\left(\left|a_{n}\right|+\left|b_{n}\right|\right)\right)<\left|z_{1}-z_{2}\right|(1+k)
\end{aligned}
$$

and

$$
\left|f\left(z_{1}\right)-f\left(z_{2}\right)\right|>\left|z_{1}-z_{2}\right|\left(1-\sum_{n=1}^{\infty} n^{p}\left(\left|a_{n}\right|+\left|b_{n}\right|\right)\right)>\left|z_{1}-z_{2}\right|(1-k) .
$$

For $z \in \mathbb{C} \backslash \tilde{\mathbb{U}}$ we have

$$
\left|F_{z}\right|=\left|1+\sum_{n=1}^{\infty} n \overline{b_{n}} z^{n-1}\right| \geq 1-\sum_{n=1}^{\infty} n\left|b_{n}\right| \geq 1-\sum_{n=1}^{\infty} n^{p}\left(\left|a_{n}\right|+\left|b_{n}\right|\right) \geq 1-k>0
$$


and

$$
\begin{aligned}
\left|\mu_{F}(z)\right| & =\left|\frac{F_{\bar{z}}}{F_{z}}\right|=\left|\frac{\sum_{n=1}^{\infty} n a_{n} \overline{z^{n-1}}}{1+\sum_{n=1}^{\infty} n \overline{b_{n}} z^{n-1}}\right| \leq \frac{\sum_{n=1}^{\infty} n^{p}\left|a_{n}\right|}{1-\sum_{n=1}^{\infty} n^{p}\left|b_{n}\right|} \\
& \leq \frac{\sum_{n=1}^{\infty} n^{p}\left|a_{n}\right|}{1-k+\sum_{n=1}^{\infty} n^{p}\left|a_{n}\right|}=1-\frac{1-k}{1-k+\sum_{n=1}^{\infty} n^{p}\left|a_{n}\right|} \leq k<1 .
\end{aligned}
$$

This means that

$$
J_{F}(z)=\left|F_{z}\right|^{2}-\left|F_{\bar{z}}\right|^{2}=\left|F_{z}\right|^{2}\left(1-\left|\mu_{F}(z)\right|^{2}\right)>0, \quad z \in \mathbb{C} \backslash \tilde{\mathbb{U}} .
$$

By the above considerations, $F$ given by (10) is continuous and locally univalent on $\mathbb{C}$ and $\lim _{z \rightarrow \infty} f(z)=\infty$. Hence (see [6], Theorem 2.7.2) the mapping $F$ is a sense-preserving homeomorphism of $\overline{\mathbb{C}}$ onto itself. Its complex dilatation satisfies

$$
\left|\mu_{F}(z)\right|<k<1
$$

for any $z \in \mathbb{C} \backslash \mathbb{T}$. Since $\mathbb{T}$ is a removable set for $F$ (see [4], p. 44), it follows that $F$ is quasiconformal in the whole plane.

\section{REFERENCES}

[1] Hengartner W., Schober G., Univalent harmonic functions, Trans. Amer. Math. Soc. 299 (1987), 1-31.

[2] Jahangiri, Jay M., Harmonic meromorphic starlike functions, Bull. Korean Math. Soc. 37 (2000), No. 2, 291-301.

[3] Jahangiri, Jay M., Silverman H., Meromorphic univalent harmonic functions with negative coefficients, Bull. Korean Math. Soc. 36 (1999), No. 4, 763-770.

[4] Lehto O., Virtanen K. I., Quasiconformal Mappings in the Plane, Springer-Verlag, Berlin-Heidelberg-New York, Second Edition, 1973.

[5] Pommerenke Ch., Univalent Functions, Vandenhoeck \& Ruprecht in Göttingen, 1975.

[6] Sheil-Small T., Complex Polynomials, Cambridge University Press, 2002.

M. Gregorczyk

Institute of Mathematics

Maria Curie-Skłodowska University

20-031 Lublin

Poland

e-mail: mgregorczyk@gmail.com

Received April 26, 2009
J. Widomski

Institute of Mathematics

Maria Curie-Skłodowska University

20-031 Lublin

Poland

e-mail: jwidomski@hektor.umcs.lublin.pl 